\title{
Research on $\frac{1}{0}, \frac{0}{0}, 0^{0}$ and Complete Theorem of
} $\frac{1}{0}-\frac{1}{0}=\frac{0}{0}-0^{0}$

\section{Weiwen Cao*}

Yinchuan Elite School, Yinchuan 750002, Ningxia Hui Autonomous Region, China

*Corresponding author: Weiwen Cao, caoweiwen2008@126.com

\begin{abstract}
It is known that 0 cannot be taken as a denominator which not only leads to $\frac{1}{0}, \frac{0}{0}$, and $0^{0}$ as meaningless but also compels many functions to appear discontinuous while numerous existing formulas would be subjected to restrictions. More importantly, the research on mathematics is also restricted to a certain extent in terms of their direction. Therefore, it is of great significance to endow 0 as a denominator in perfecting the current algorithm to solve a series of problem in the mathematics field that has been persistently present for a long time, to fill in many research gaps that have been neglected, as well as to elevate mathematical research to a higher level.
\end{abstract}

Keywords: $0 ; \frac{1}{0} ; \frac{0}{0} ; 0^{0} ;$ Number of poles; Complete theorem

Publication date: June 2021; Online publication: June 30, 2021

\section{Introduction}

For a long period of time, people have insisted that zero has no reciprocal and it cannot be used as a divisor. Moreover, the use of 0 as a base or exponent has been strictly restricted in which $\frac{1}{0}, \frac{0}{0}$, and $0^{0}$ are meaningless. There seems to be a good reason to agree with these statements. First, if 0 is the divisor, the dividend is divided into zero and non-zero cases. However, if the dividend is 0 , then no matter what the quotient is, the product of the quotient and the divisor, 0 is 0 ; hence, the quotient has an infinite number of possibilities. Meaning to say, the quotient is uncertain. If the dividend is non- 0 , then the product of the quotient and the divisor, 0 is 0 no matter what the quotient is, however, the dividend itself is a non- 0 , so it is not possible. Second, a positive integer $m$ (the dividend) is divided by another positive integer $n$ (the divisor) in which $\frac{m}{n}$ means that the size of each of the dividend $m$ is divided equally into $n$ parts. So, at the time of $n=0$, the meaning of $\frac{m}{0}$ is to average out a 0 dividend which of course would not make sense. Third, in Einstein's theory of relativity, there is a specific relationship between the mass, $m$ of an object and its velocity, $v$ in which, $m=\frac{m_{0}}{\sqrt{1-\left(\frac{v}{c}\right)^{2}}}$. The faster an object is moving $(v)$, the greater its mass $(m)$ will be. As the velocity $(v)$ approaches infinity which is the speed of light $(c)$, the denominator, $\sqrt{1-\left(\frac{v}{c}\right)^{2}}$ of the formula from infinity approaches 0 , then the mass $(m)$ of the object approaches to $+\infty$. Once an object reaches the speed of light $(v=c)$, immediately $\sqrt{1-\left(\frac{v}{c}\right)^{2}}=0$, then the mass of the object would be, $m=\frac{m_{0}}{0}$ which is obviously counterintuitive. In other words, a statically massive object $m_{0} \neq$ 0 cannot reach the speed of light. In regard to the photons that make up light, the static mass in relativity 
$m_{0}=0$. Only objects with a static mass of 0 can reach the speed of light. 0 cannot be a divisor.

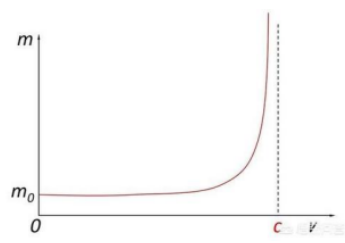

Figure 1.

While these arguments seem sound enough, they are not irrefutable. In regard to the first reason, when there are many possible results according to the algorithm, one of them can be determined as the final result. For example, since the length of 0 is 0 , its direction is arbitrary. That means that the 0 angle with any nonzero vector could be any angle but specifying that 0 is parallel to any non-zero vector ${ }^{[1]}$ The second reason is not an excellent reason. If the second reason is true, then things like $\frac{-1}{-2}, \frac{-3}{4}, \frac{5}{-6}, \frac{7}{8.9}, \frac{\pi}{10}, \frac{0}{e}$, and $i^{2}=$ -1 would be meaningless. In the third argument, although seemingly enigmatic, it is in fact contradictory. Since $v=c$, the velocity of the photon is satisfied and relativity $m_{0}=0$ also postulates that of the static mass of the photon. Is it true that the denominator of the default mass increment formula $\sqrt{1-\left(\frac{v}{c}\right)^{2}}$ can be 0 and $\frac{0}{0}$ but exists? All in all, there are three good reasons for zero not to be a divisor and none of which stand up to scrutiny with detailed examination. However, under the misdirection of these traditional, narrow-minded, and obscure views, people consistently insist that 0 cannot be used to divide and therefore, there is no point of $\frac{0}{0}$. In addition to that, because $0^{0}=0^{1-1}=0^{1} \times 0^{-1}=0 \times \frac{1}{0}=\frac{0}{0}$, so $0^{0}$ makes no sense. This leads to certain disjunctions in $y=\tan x, y=\frac{1}{x}, y=x^{0}, y=0^{x}$, and functions of the same type. If proper meanings are given to $\frac{1}{0}, \frac{0}{0}$, and $0^{0}$ in addition to the improvement of the algorithm, not only would the above disjunctions be eliminated but also, the algorithm could be perfected.

It is known that theta $C_{n}^{0}=C_{n}^{n}=1$ but what is $C_{0}^{0}$ ? Since $C_{n}^{m}=\frac{n !}{m !(n-m) !}$, so when $n>0$ and $m=0, C_{n}^{0}=\frac{n !}{0 !(n-0) !}=\frac{n !}{0 ! \cdot n !}=\frac{1}{0 !}=1$ and $0 !=1$. These are important result because $n !=$ $n \times(n-1) \times(n-2) \times \cdots \times 3 \times 2 \times 1$ and that the 0 ! factorial formula does not work. According to the above formula, $C_{0}^{0}=\frac{0 !}{0 ! \times(0-0) !}=\frac{0 !}{0 ! \times 0 !}=\frac{1}{1 \times 1}=\frac{1}{1}=1$, thereby $C_{0}^{0}=1$. In fact, this is a far more important than the conclusion of $0 !=1$ because it would be an important addition to the binomial theorem!

According to the binomial theorem, $(a+b)^{n}=C_{n}^{0} a^{n}+C_{n}^{1} a^{n-1} b^{1}+\cdots+C_{n}^{k} a^{n-k} b^{k}+\cdots+C_{n}^{n} b^{n}$. When $n=0, a=1, b=-1,(a-b)^{n}=(1-1)^{0}=C_{0}^{0}(1)^{0}(-1)^{0}=1 \times 1 \times 1=1=0^{0}$, so $0^{0}=$ 1 and because $0^{0}=\frac{0}{0}$; hence, $\frac{1}{0}, \frac{0}{0}$, and $0^{0}$ must exist and have meaning. Of course, the same conclusion can also be deduced from the equiproportional property whereby if $\frac{a}{b}=\frac{c}{d}=\frac{e}{f}=\cdots=\frac{m}{n}$, then $\frac{a+c+e+\cdots+m}{b+d+f+\cdots+n}=\frac{a}{b}$. So, if $\frac{1}{1}=\frac{-1}{-1}$, then $\frac{1-1}{1-1}=\frac{0}{0}=\frac{1}{1}=\frac{-1}{-1}=1$ and $\frac{0}{0}=1$.

If $\frac{1}{0}$ does exist, what does that mean? For the inverse proportional function of $y=\frac{1}{x}$, when $x \rightarrow 0$, $\lim _{x \rightarrow 0^{+}} \frac{1}{x}=+\infty$ and $\lim _{x \rightarrow 0^{-}} \frac{1}{x}=-\infty$. However, going along this line, the question is when $x=0$, what is the value of $y=\frac{1}{x}$ then? It is generally believed that $x$ can only closely approach 0 but would never reach it. ${ }^{[2]}$ There is a sense of unreachable indignation whereby if $x$ can easily travel across the number line to get to any number, why then it cannot reach 0 ? It is as if a spaceship is built with the capacity to instantly 
travel through the entire universe with ease and reach any celestial body for exploration, but it is unable to get into the cabin to launch itself. It can only get infinitely closer. It is therefore natural that one should be given the right to divide, so that the denominator can be zero and to give a sense of "polar number" - "the greatest number." $\frac{1}{0}$ is the end of what is known as a number, and there is no number greater than that. In order to express the values of $\frac{1}{0}, \frac{0}{0}$, and $0^{0}$ as well as their relations, the equation $\frac{1}{0}-\frac{1}{0}=\frac{0}{0}-0^{0}$ can be used. This equation and its related contents are called "complete theorem." After the introduction of the "complete theorem," the following important issues will be discussed.

\section{Re-understanding point, line, plane, and body as well as their mutual relations through the concept of "polar number"}

As understood, a line is made up of an infinite number of points while a plane is made up of an infinite number of lines and a body is made up of an infinite number of faces. However, there are a few problems with this statement. For example, there will always be some distance $\sigma$ between any two points on a line but of course, dichotomy can be used to reduce this distance. After partitioning $n$ times, the distance between two points becomes $\frac{\sigma}{2^{n}}$ with whatever value of $n$, even if $n \rightarrow+\infty, \frac{\sigma}{2^{+\infty}}>0$. This means that the line is not continuous, but it is an infinite number of points in discontinuity. On the other hand, since a point has no diameter, area, or volume, a line also should not have length, area, or volume. Is it not a paradox that surfaces and bodies do not have areas and volumes? Although in actual learning, education, and research, the concepts of length, area, and volume are formed by "accumulating" points, lines, and surfaces on paper or on the blackboard. This is because the points, lines, and surfaces drawn by people with tools do have a certain diameter, area, and volume. If an atom has no diameter, area, or volume, then bodies would also have no length, area, or volume. Unfortunately, points, as they are called in mathematics have no diameter, area, or volume.

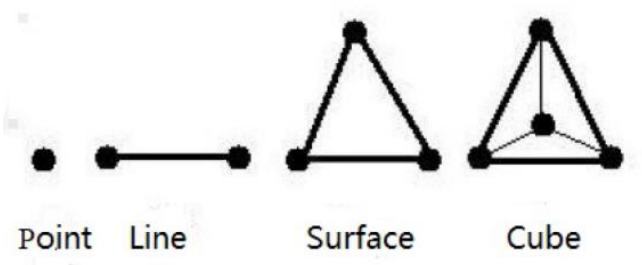

Figure 2.

For a line segment of a length of 1 , it will have $\frac{1}{0}$ points at most. The distance between two adjacent points is $\sigma=\frac{1}{\frac{1}{0}}=1 \times 0=0$ and the area and the volume of that "line segment" are $s=0 \times \frac{1}{0}=1$ and $v=0 \times \frac{1}{0}=1$, respectively. The area of a square with sides that is 1 in length is $S=1 \times 1=1$ and the volume of a cube with sides that is 1 in length is $V=1 \times 1 \times 1=1$. For a line segment that is $l(l \geq 1)$ in length and whose area as well as volume are also numerically equivalent to $l$, the "line segment" will have $l \cdot \frac{1}{0}=\frac{1}{0}$ points at most. When $0 \leq l<1$, its area and volume are numerically equivalent to $l$; hence, there will be at most $l \cdot \frac{1}{0}=\frac{l}{0}$ points ( $l$ is not cancellable; see the algorithm for polar numbers below) on 
the "line segment." In this way, the "accumulation" of the length, area and volume of the subsequent lines, planes, and bodies is prepared. However, there is a surprising conclusion in which $\frac{1}{0}$ is the maximum length a line (including a ray) may reach. Meanwhile, points on the line are no more than $\frac{1}{0}$, so the distance between two adjacent points is $\sigma=\frac{\frac{1}{0}}{\frac{1}{0}}=1$. This means that what is commonly referred to as an infinite number of points on a line is actually a finite part of a line that is regarded as a "line segment" in the study of a line. This also shows that the operation of $\frac{1}{0}$ is irreversible. For example, one cannot claim that a graph with area of 10 has $\frac{10}{0}$ points. Although it does have $\frac{1}{0}$ points but the area is not $S=0 \times \frac{1}{0}=1$; instead, it is, $S=10 \times 1=10$.

\section{Re-understanding the discontinuity point after the introduction of the concept of polar number}

For functions of $y=\tan x, y=\frac{1}{x}, y=x^{0}, y=0^{x}$, and other similar types of functions, it is assumed that their analytic expression is $y=f(x)$ and a few disjunctions are usually drawn on their graphs. Generally, discontinuity points are divided into two categories. If $x_{0}$ is the discontinuity point of a function $f(x)$, but both the left limit $f\left(x_{0}{ }^{-}\right)$and the right limit $f\left(x_{0}{ }^{+}\right)$exist, then $x_{0}$ is known as the discontinuity point of the first kind of function $f(x)$. Any discontinuity that is not a discontinuity of the first kind is called a discontinuity of the second kind. In the first kind, those with equal left and right limits are called removable discontinuities while those with unequal limits are called jump discontinuities. The infinite and oscillating discontinuities are obviously discontinuities of the second kind. ${ }^{[2]}$

Suppose that one of the discontinuity points is $x_{0}$, and there is a definition in a neighborhood of $x_{0}$ which is $\lim _{x \rightarrow x_{0}} f(x)=y_{0}$. If a value $y_{0}$ is assigned to this function $y=f\left(x_{0}\right), \lim _{\Delta x \rightarrow 0} \Delta y=\lim _{\Delta x \rightarrow 0}\left[f\left(x_{0}+\right.\right.$ $\left.\Delta x)-f\left(x_{0}\right)\right]=0$ must exist. From this we can see that $y=f(x)$ is continuous at $x=x_{0} \cdot{ }^{[2]}$ With the same token, the problem of other disjunctions will be solved. For example, the function $y=\frac{x^{2}-1}{x-1}$ is undefined at $x=1$ hence, the function is discontinuous at $x=1$. But as $x$ approaches 1 , the limit of the function on the left and the right of $x=1$ exist and are equal, which is $\lim _{x \rightarrow 1} \frac{x^{2}-1}{x-1}=\lim _{x \rightarrow 1}(x+1)=2$. If the additional definition is: let $x=1$ and $y=2$, then the given function is continuous at $x=1$, hence, $x=1$ is the removable discontinuity point of the function. ${ }^{[2]}$

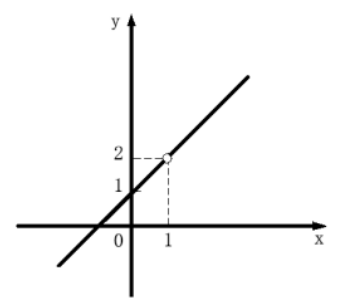

Figure 3. 


\section{Possibility of directly replacing the denominator in formulas with 0 since 0 can be the denominator}

Although $\frac{1}{0}$ has been given a proper meaning, it does not mean that the denominator in the formula can be directly replaced by 0 . This is because it is either impossible to obtain "formulas" with that denominator according to the improved algorithm or the "formulas" would be meaningless. A few examples are shown below.

Example 1. For an equation in the intercept form $\frac{x}{a}+\frac{y}{b}=1$, the line passes through the origin when $a=b=0$. If the denominator is directly replaced with $0, \frac{x}{0}+\frac{y}{0}=1$ is obtained. However, for a line that passes through the origin (suppose $k \geq 1$ ), $y=k x \Rightarrow \frac{y}{0}=\frac{k x}{0} \Rightarrow \frac{k x}{0}-\frac{y}{0}=0 \Rightarrow \frac{x}{0}-\frac{y}{0}=0$, which is not the same as $\frac{x}{0}+\frac{y}{0}=1$. When the line is the $y$ axis, the slope $k=\frac{1}{0}$. If $y=k x$ is fit in, $y=\frac{1}{0} \cdot x$ is obtained. Therefore, when $x=0, y=\frac{1}{0} \times 0=1$ which is obviously false, because $y$ is not the function $x$. When $x=0, x$ corresponds to an infinite number of values of $y$, so the analytic expression of the function should be $x=0$.

Example 2. In the case of the tolerance formula $d=\frac{a_{n}-a_{m}}{n-m}$; when $m=n$, then $d=\frac{a_{n}-a_{n}}{n-n}=\frac{0}{0}=1$ which is obviously wrong. This is because there is no "tolerance" between a number and itself in which tolerance requires at least two numbers. However, it is possible to admit that an individual number has a tolerance in which of course the tolerance can only be 0 . Hence, what is the problem then? We know that, $\left\{\begin{array}{l}a_{n}=a_{1}+(n-1) d \\ a_{m=m}=a_{1}+(m-1) d\end{array} \Rightarrow a_{n}-a_{m}=0+(n-m) d \Rightarrow a_{n}-a_{m}-0=(n-m) d \Rightarrow \frac{a_{n}-a_{m}}{n-m}-\frac{0}{n-m}=\right.$ $d \stackrel{n=m}{\Rightarrow} \frac{a_{n}-a_{n}}{n-n}-\frac{0}{n-n}=d \Rightarrow \frac{0}{0}-\frac{0}{0}=d \Rightarrow 1-1=d \Rightarrow d=0$. What is noticeable is $a_{n}-a_{m}-0=(n-$ $m) d \Rightarrow \frac{a_{n}-a_{m}-0}{n-m}=d \stackrel{n=m}{\Rightarrow} \frac{a_{n}-a_{n}-0}{n-n}=d \Rightarrow \frac{0}{0}=d \Rightarrow d=1$, which is obviously wrong. That is because $\frac{0}{0}-\frac{0}{0} \neq \frac{0-0}{0}$, which means comparing $\frac{0-0}{0}$ to $\frac{0}{0}-\frac{0}{0}$, there are "obscures" in the calculation of $\frac{0}{0}$.

Example 3. In view of the theorem of sine $\frac{a}{\sin A}=\frac{b}{\sin B}=\frac{c}{\sin C}=2 R ; A, B$, and $C$ respectively represent the three angles of $\triangle A B C$. Any of these angles would make the sine law meaningless and other data would change if one of the angles is 0 .

Example 4. In regard to the formula concerning distance from point and line $d=\frac{|A x+B y+C|}{\sqrt{A^{2}+B^{2}}}$; if $A=$ $B=0$, then $C=0$. The equation of the line is meaningless, and there will be no distance between the point and the line.

\section{Making sense of the logarithmic function $y=\log _{a} x,(a>0)$, when $x \leq 0$}

In the logarithm function $y=\log _{a} x,(a>0), x$ may take any value. When $x<0, y=\log _{a} x=$ $\log _{a}(-x) \cdot(-1)=\log _{a}(-x) \cdot i^{2}=\log _{a}(-x)+\log _{a} i^{2}=\log _{a}(-x)+2 \log _{a} i$. When $x=0, y=\log _{a} x=\log _{a} 0$; when $a>1, y=\log _{a} 0=-\frac{1}{0}$; when $0<a<1, y=\log _{a} 0=\frac{1}{0}$; when $a=1, y=\log _{a} 0$; which does not exist.

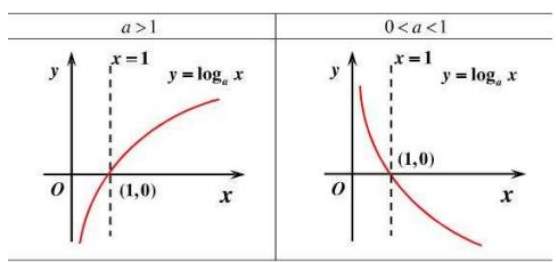

Figure 4. 


\section{Viewing the static mass of photons $m_{0}=0$ in Einstein's theory of relativity}

In the mass increment equation $m=\frac{m_{0}}{\sqrt{1-\left(\frac{v}{c}\right)^{2}}}$, the velocity of the photon does satisfy $v=c$. When photons move, do they have no mass? Of course not! As mentioned above, only with static mass of photons $m_{0}=$ 0 , only then can the dynamic mass of the photon of $m=\frac{m_{0}}{0}=\frac{0}{0}=1$ exist.

7. Reasonability of the stipulation that $\overrightarrow{0}$ is parallel to any non-zero vector when the direction of $\overrightarrow{0}$ is arbitrary and determining the angle between them $\left(\begin{array}{lll}0 & \text { or } \pi\end{array}\right)$ as well as the relationship between $\overrightarrow{0}$ and $\overrightarrow{0}$

It is known that $\cos \theta=\frac{\vec{a} \vec{b}}{|\vec{a}||\vec{b}|}=\frac{x_{1} x_{2}+y_{1} y_{2}}{\sqrt{x_{1}^{2}+y_{1}^{2}} \sqrt{x_{2}^{2}+y_{2}^{2}}}$, where $\vec{a}=\left(x_{1}, y_{1}\right)$ and $\vec{b}=\left(x_{2}, y_{2}\right)$. Assuming $\vec{a} \neq \overrightarrow{0}$, $\vec{b}=\overrightarrow{0}, \cos \theta=\frac{x_{1} \cdot 0+y_{1} \cdot 0}{\sqrt{x_{1}^{2}+y_{1}^{2}} \cdot \sqrt{0^{2}+0^{2}}}=\frac{0}{\sqrt{x_{1}^{2}+y_{1}^{2}} \cdot 0}=\frac{0}{0}=1$ can be obtained, so $\theta=0$. Not only is $\overrightarrow{0}$ parallel to any non-zero vector, but it is also at a zero angle. When $\vec{a}=\vec{b}=\overrightarrow{0}, \cos \theta=\frac{0 \times 0+0 \times 0}{\sqrt{0^{2}+0^{2}} \times \sqrt{0^{2}+0^{2}}}=\frac{0}{0}=1$, so $\theta=0 . \overrightarrow{0}$ and $\overrightarrow{0}$ are not only parallel but they are also at an angle of 0 .

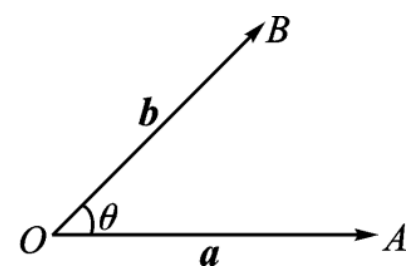

Figure 5 .

8. Applicability of the sum formula of geometric series $S_{n}=\frac{a_{1}\left(1-q^{n}\right)}{1-q}$ in regard to the common ratio $q=1$

When $q=1$, the sum formula of geometric series $S_{n}=\frac{a_{1}\left(1-q^{n}\right)}{1-q}$ is still applicable and it can even be applied to the constant based on 0 . When $q=1$, according to the passage, $S_{n}=\frac{a_{1}\left(1-q^{n}\right)}{1-q}$, it is known as follows:

$$
\begin{aligned}
& S_{1}=\frac{a_{1}\left(1-q^{1}\right)}{1-q}=\frac{a_{1}(1-q)}{1-q}=a_{1} \\
& S_{2}=\frac{a_{1}\left(1-q^{2}\right)}{1-q}=\frac{a_{1}(1-q)(1+q)}{1-q}=a_{1}(1+q)=a_{1}(1+1)=2 a_{1} \\
& S_{3}=\frac{a_{1}\left(1-q^{3}\right)}{1-q}=\frac{a_{1}(1-q)\left(1^{2}+1 \cdot q+q^{2}\right)}{1-q}=a_{1}\left(1^{2}+1 \cdot q+q^{2}\right)=a_{1}\left(1^{2}+1 \times 1+1^{2}\right)=3 a_{1} \\
& S_{4}=\frac{a_{1}\left(1-q^{4}\right)}{1-q}=\frac{a_{1}\left(1-q^{2}\right)\left(1+q^{2}\right)}{1-q}=\frac{a_{1}(1-q)(1+q)\left(1+q^{2}\right)}{1-q} \\
& =a_{1}(1+q)\left(1+q^{2}\right)=a_{1}(1+1)\left(1+1^{2}\right)=a_{1}(1+1)(1+1)=a_{1} \times 2 \times 2=4 a_{1}
\end{aligned}
$$




$$
\begin{aligned}
& S_{5}=\frac{a_{1}\left(1-q^{5}\right)}{1-q}=\frac{a_{1}(1-q)\left(1^{4}+1^{3} \cdot q+1^{2} \cdot q^{2}+1 \cdot q^{3}+q^{4}\right)}{1-q} \\
& =a_{1}\left(1^{4}+1^{3} \cdot q+1^{2} \cdot q^{2}+1 \cdot q^{3}+q^{4}\right)=a_{1}\left(1^{4}+1^{3} \times 1+1^{2} \times 1^{2}+1 \times 1^{3}+1^{4}\right)=5 a_{1} \\
& S_{6}=\frac{a_{1}\left(1-q^{6}\right)}{1-q}=\frac{a_{1}\left(1-q^{3}\right)\left(1+q^{3}\right)}{1-q}=\frac{a_{1}(1-q)\left(1^{2}+1 \cdot q+q^{2}\right)\left(1+q^{3}\right)}{1-q} \\
& =a_{1}\left(1^{2}+1 \cdot q+q^{2}\right)\left(1+q^{3}\right)=a_{1}\left(1^{2}+1 \times 1+1^{2}\right)\left(1+1^{3}\right)=a_{1}(1+1+1)(1+1)=6 a_{1}
\end{aligned}
$$

By the same token, $S_{n}=n a_{1}$.

The above evaluation method is very similar to the method of obtaining the limit in higher mathematics. For example, the calculation of $\lim _{x \rightarrow 3} \frac{x-3}{x^{2}-9}$ is $\lim _{x \rightarrow 3} \frac{x-3}{x^{2}-9}=\lim _{x \rightarrow 3} \frac{x-3}{(x-3)(x+3)}=\lim _{x \rightarrow 3} \frac{1}{(x+3)}=\frac{\lim _{x \rightarrow 3} 1}{\lim _{x \rightarrow 3}(x+3)}=\frac{1}{3+3}=\frac{1}{6}{ }^{[2]}$ Often, this kind of problems have three following characteristics. The first, when $x \rightarrow a$, both the numerator and denominator of the fraction tend to be 0 . Second, in the neighborhood of $x=x_{0}$, the derivatives of both the numerator and the denominator exist. Third, the fraction exists at the limit of $x=$ $x_{0} \cdot{ }^{[2]}$ These characteristics are very similar to the requirements of L'Hopital's rule only because of the nonzero denominators. However, L'Hopital's rule is more demanding. Therefore, this kind of problem can be well solved by the L'Hopital's rule in which the above equation is used as an example.

Using L'Hopital's rule, the sum formula of geometric series is restudied in which $\lim _{q \rightarrow 1} S_{n}=$ $\lim _{q \rightarrow 1} \frac{a_{1}\left(1-q^{n}\right)}{1-q}=\lim _{q \rightarrow 1} \frac{\left[a_{1}\left(1-q^{n}\right)\right]^{\prime}}{(1-q)^{\prime}}=\lim _{q \rightarrow 1} \frac{a_{1}\left(0-n q^{n-1}\right)}{0-1}=\lim _{q \rightarrow 1} n a_{1} q^{n-1}=n a_{1} \cdot 1^{n-1}=n a_{1}$. Strangely, $S_{n}=$ $\frac{a_{1}-a_{n} q}{1-q}$ is not applicable to the above algorithm. This is because $S_{n}=\frac{a_{1}\left(1-q^{n}\right)}{1-q}=\frac{a_{1}-a_{1} q^{n}}{1-q}=$ $\frac{a_{1}-\left(a_{1} q^{n-1}\right) q}{1-q}=\frac{a_{1}-a_{n} q}{1-q}$ in which an obscure of the process $q$ occurred using L'Hopital's rule.

Finally, the constant series of terms are examined whereby the summation formula also applies. $0 a_{1}=$ $a_{2}=\cdots=a_{n}=0 q=\frac{a_{k+1}}{a_{k}}=\frac{0}{0}=1 \quad(0<k<n$ 且 $k \in N) \lim _{\substack{a_{n} \rightarrow 0 \\ q \rightarrow 1}} S_{n}=\lim _{a_{n} \rightarrow 0} n a_{1}=n \cdot 0=0$

9. Possibility of using the root formula $x=\frac{-b \pm \sqrt{b^{2}-4 a c}}{2 a}$ to solve the quadratic function of one variable $a x^{2}+b x+c=0$, when $a=0, b \neq 0,0+b x+c=0 \Rightarrow b x=-c \Rightarrow x=-\frac{c}{b}$

When $a=0 ; a x^{2}+b x+c=0 \Rightarrow a x^{2}+b x=-c \Rightarrow x^{2}+\frac{b}{a} x=-\frac{c}{a} \Rightarrow x^{2}+\frac{b}{a} x+\left(\frac{b}{2 a}\right)^{2}=-\frac{c}{a}+$ $\left(\frac{b}{2 a}\right)^{2} \Rightarrow\left(x+\frac{b}{2 a}\right)^{2}=\frac{b^{2}-4 a c}{4 a^{2}}$.

When $\quad b>0 \quad ; \quad x+\frac{b}{2 a}=+\sqrt{\frac{b^{2}-4 a c}{4 a^{2}}} \Rightarrow x=-\frac{b}{2 a}+\sqrt{\frac{b^{2}-4 a c}{4 a^{2}}}=-\frac{b}{2 a}+\frac{\sqrt{b^{2}-4 a c}}{2 a}=\frac{-b+\sqrt{b^{2}-4 a c}}{2 a}=$ $\lim _{a \rightarrow 0} \frac{\left(-b+\sqrt{b^{2}-4 a c}\right)^{\prime}}{(2 a)^{\prime}}=\lim _{a \rightarrow 0} \frac{\frac{-4 c}{2 \sqrt{b^{2}-4 a c}}}{2}=\lim _{a \rightarrow 0} \frac{-c}{\sqrt{b^{2}-4 a c}}=\frac{-c}{\sqrt{b^{2}-0}}=-\frac{c}{b}$.

When $\quad b<0 \quad ; \quad x+\frac{b}{2 a}=-\sqrt{\frac{b^{2}-4 a c}{4 a^{2}}} \Rightarrow x=-\frac{b}{2 a}-\sqrt{\frac{b^{2}-4 a c}{4 a^{2}}}=-\frac{b}{2 a}-\frac{\sqrt{b^{2}-4 a c}}{2 a}=\frac{-b-\sqrt{b^{2}-4 a c}}{2 a}=$ $\lim _{a \rightarrow 0} \frac{\left(-b-\sqrt{b^{2}-4 a c}\right)^{\prime}}{(2 a)^{\prime}}=\lim _{a \rightarrow 0} \frac{-\frac{-4 c}{2 \sqrt{b^{2}-4 a c}}}{2}=\lim _{a \rightarrow 0} \frac{c}{\sqrt{b^{2}-4 a c}}=\frac{c}{\sqrt{b^{2}-0}}=-\frac{c}{b}$. 
It should be noted that $\left(-\frac{b}{2 a}, \frac{4 a c-b^{2}}{4 a}\right)$ is applicable if $a=0$ because there is no axis of symmetry, $x=-\frac{b}{2 a}$ and vertex ordinate, $y=\frac{4 a c-b^{2}}{4 a}$; hence, substituting in $a=0$ cannot be done.

\section{Finding the value of the function $y=\sin \frac{1}{x}$ when $x=0$}

As $x$ approaches 0 from the left and right, the image of the function $y=\sin \frac{1}{x}$ oscillates violently which is traditionally known as the oscillating function while $x=0$ is known as the oscillation discontinuity point of the function $y=\sin \frac{1}{x}$. However, the oscillation discontinuity point is neither a jump discontinuity point nor an infinite discontinuity point. In other words, it is a removable discontinuity point due to the absence of a definition when the function $x=0$ is continuous. Hence, if $y=\sin \frac{1}{x}$ is an odd function when $x=0, y=\sin \frac{1}{0}=0$.

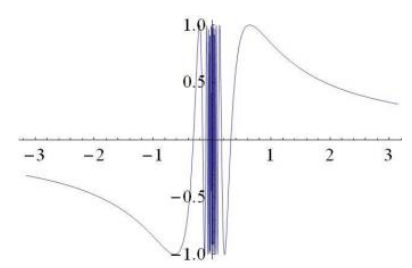

Figure 6.

\section{Finding the value of the inverse proportional function $y=\frac{1}{x}$ when $x=0$}

In the inverse proportional function $y=\frac{1}{x}$, the value of the function is $-\frac{1}{0}$ when $x$ moves from the left to 0 ; and is $\frac{1}{0}$ when $x$ moves from the right to 0 . Therefore, $x=0$ is conventionally known as the infinite break point of the function $y=\frac{1}{x}$. What then is the value of the function $y=\frac{1}{x}$, when $x=0$ ? When the function values on both sides of the original discontinuity point are different and the definition of the discontinuity point is missing, the function value at the original discontinuity point would be subjected to the results of $x$ arriving at the original discontinuity point from the right side which is when $x=0, y=$ $\frac{1}{x}=\frac{1}{0}$. Similarly, when $x=\frac{\pi}{2}+k \pi$ and $k \in Z$ where the values on the left and right $k \in Z$ are negative, and moving toward plus or minus infinity, the values of the tangent function $y=\tan x=-\frac{1}{0}$.

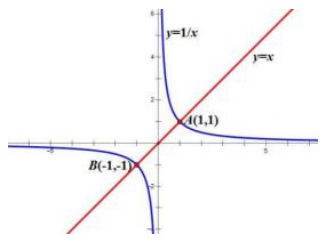

Figure 7.

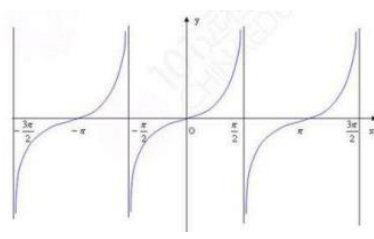

Figure 8 .

12. Possibility of $P(A)$ to be 0 in the conditional probability, $P(B \mid A)=\frac{P(A B)}{P(A)}$ For conditional probability $P(B \mid A)=\frac{P(A B)}{P(A)}, P(A)=0$ still applies. For example, among five multiple questions, there are three science questions and two liberal arts questions. By setting "draw a comprehensive topic of arts and science the first time" as event $A$, "draw a science topic the first time" as event $B$, and "draw a science topic both the first and the second time" as event $A B$, if two questions are 
drawn in order, the conditional probability is $P(B \mid A)=\frac{P(A B)}{P(A)}=\frac{P(A) \cdot P(B)}{P(A)}=$ $\lim _{P(A) \rightarrow 0} \frac{[P(A) \cdot P(B)]^{\prime}}{[P(A)]^{\prime}}=\frac{P(B)}{1}=\frac{3}{5} \times \frac{2}{4}+\frac{2}{5} \times \frac{3}{4}=\frac{3}{5} \cdot[3]$

13. Possibility of 0 as the image area of the normal distribution function of $f(x)=\frac{1}{\sqrt{2 \pi} \sigma} e^{-\frac{(x-\mu)^{2}}{2 \sigma^{2}}}$, $x \in(-\infty,+\infty)$ when $\sigma=0$

In the normal distribution function $f(x)=\frac{1}{\sqrt{2 \pi} \sigma} e^{-\frac{(x-\mu)^{2}}{2 \sigma^{2}}}$ when $x \in(-\infty,+\infty), \mu$ and $\sigma$ represent the mean and standard deviation of the population respectively. The curve is symmetric about the line $x=$ $\mu$ and reaches its highest point in case of $x=\mu$. When $\mu$ is certain, the shape of the curve is determined by $\sigma$. Larger $\sigma$ results in a "shorter and fatter" curve which means that the distribution of the population is more dispersed. The smaller the $\sigma$ is, the "thinner and higher" the curve is, which indicates that the distribution of the population is more concentrated. When $\sigma$ is the same, the position of the curve is determined by $\mu$. The curve is above the $x$ axis and the area that covers down to the $x$ axis is 1 . When $\sigma=0$ and $x=\mu=0, f(\mu)=\frac{1}{\sqrt{2 \pi} \cdot 0} e^{-\frac{(\mu-\mu)^{2}}{2 \times 0^{2}}}=\frac{1}{0} \cdot e^{\frac{0}{0}}=\frac{1}{0} \cdot e^{1}=\frac{1}{0}$. The area enclosed by the curve and axis $x$ is: $S=0 \times \frac{1}{0}=1$.

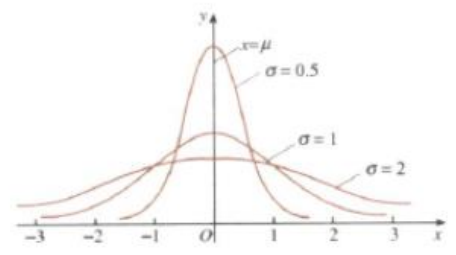

Figure 9.

\section{Possibility of the graph of function $y=0 \cdot x$ being identical with line $y=0$}

Generally, 0 times anything equals to 0 . However, the concept of polar number has been introduced and $0 \times \frac{1}{0}=1$. When $x_{1}=\frac{1}{2} \times \frac{1}{0}, x_{2}=\frac{1}{3} \times \frac{1}{0}, x_{3}=\frac{1}{4} \times \frac{1}{0}$, and so on, what does $y$ equal to then? By substitute the following values into the above equations respectively; $y_{1}=0 \times \frac{1}{2} \times \frac{1}{0}=\frac{1}{2}, y_{2}=$ $0 \times \frac{1}{3} \times \frac{1}{0}=\frac{1}{3}, y_{3}=0 \times \frac{1}{3} \times \frac{1}{0}=\frac{1}{3}$, and so forth, a number which is equivalent to $\frac{1}{0}$ can be seen. When $x=\infty, y=0 \times \infty=0 \cdot a \cdot \frac{1}{0}=a$ instead of $y=0 \times \infty=0$ since $\infty$ can be regarded as a number of the same magnitude in which $\infty=a \cdot \frac{1}{0}, 0<a<1$.

Similarly, imagine there is a point $A$ on a line segment and a point is randomly selected of this line segment; what then is the probability that point $A$ is selected? According to the calculation method of the geometric schema, the length of point $A$ is 0 while the length of the line segment is $l$, then the probability of selecting point $A$ is $P(A)=\frac{0}{l}=0$, which is obviously wrong. This is because $P(A)$ is small, but it exists in the line segment. So, how can this problem be understood? If the length of the line segment is short, $l=\frac{1}{a \cdot \frac{1}{0}}=\frac{1}{\frac{a}{0}}(0 \leq a \leq 1)$ (Note: $\frac{1}{\frac{a}{0}} \neq \frac{0}{a}$, see the algorithm of "pole number"), then $P(A)=\frac{0}{l}=\frac{0}{\frac{1}{\frac{a}{0}}}=$ $0 \times \frac{a}{0}=a$. If the length of the line segment is longer, $l=a \cdot \frac{1}{0}=\frac{a}{0}(0<a), P(A)=\frac{0}{l}=\frac{0}{\frac{a}{0}}=0$. After 
the introduction of the concept of "polar number," some of the original algorithms begin to be applicable. Alike the introduction of relativity in physics, the laws of motion in a slower world no longer apply to a faster world.

\section{Possibility of power function $y=x^{0}$ being identical with the graph of line $y=1$}

For power functions $y=x^{0}$, there is always $\ldots=(-3)^{0}=(-2)^{0}=(-1)^{0}=1=(1)^{0}=(2)^{0}=(3)^{0} \ldots$ Except when $x=0$, the function values corresponding to each point are 1 . When $x=0, y=0^{0}=1$ in which the graph is the same as the line $y=1$.

\section{Conclusion}

All in all, the introduction of the "complete theorem" is extremely necessary. It not only solves series of problems that have troubled the mathematical field for a long time, but also fills in many research gaps that have been neglected. The special properties of "polar number" and its algorithm have brought about new understandings and enlightenments to the study of mathematics as well as expanded mathematical studies into a wider field.

\section{Disclosure statement}

The author declares no conflict of interest.

\section{References}

[1] 2007, Institute of curriculum and textbooks, Mathematics Compulsory 4, People's Education Press, Beijing.

[2] 2007, Department of mathematics, Tongji University, Advanced Mathematics 6th Ed, Higher Education Press, Beijing.

[3] 2009, Institute of curriculum and textbooks, Mathematics elective 2-3. People's Education Press, Beijing. 